\title{
Quantitative Rotor Broken Bar Evaluation in Double Squirrel Cage Induction Machines under Dynamic Operating Conditions
}

\author{
Y. Gritli ****, A. O. Di Tommaso **, R. Miceli **, IEEE, Member, \\ C. Rossi *, IEEE, Member, and F. Filippetti *, IEEE, Member. \\ * University of Bologna, Italy. \\ Dipartimento di Ingegneria dell'Energia Elettrica e dell'Informazione «Guglielmo Marconi». \\ E-mail: yasser.gritli@unibo.it, fiorenzo.filippetti@unibo.it, claudio.rossi@unibo.it . \\ ** University of Palermo, Italy. \\ Department of Electrical, Electronic and Telecommunication Engineering, \\ Chemical Technologies, Automatics and Mathematical Models. \\ E-mails: ditommaso@,dieet.unipa.it, rosario.miceli@unipa.it. \\ *** University of Tunis El Manar, Tunisia. \\ Department of Electrical Engineering - National Engineering School of Tunis. \\ E-mail: yasser.gritli@esti.rnu.tn.
}

\begin{abstract}
Advanced monitoring techniques leading to fault diagnosis and prediction of induction machine faults, operating under non-stationary conditions have gained strength because of its considerable influence on the operational continuation of many industrial processes. In case of rotor broken bars, fault detection based on sideband components issued from currents, flux, instantaneous control or power signals under different load conditions, may fail due to the presence of inter-bar currents that reduce the degree of rotor asymmetry, especially for double squirrel cage induction motors. But the produced core vibrations in the axial direction, can be investigated to overcome the limitation of the classical technique using appropriate timefrequency analysis for these purposes. Unlike previous approaches, the presented technique is based on optimized use of the Discrete wavelet transform to overcome the limitation of classical frequency approaches under non-stationary operating conditions. The developed approach is best suited for automotive or high power traction systems, in which safe-operating and availability are mandatory. Experimental results are provided, showing the validity of the investigated technique, leading to an effective diagnosis procedure for incipient rotor broken bars in double or single cage induction machines under dynamic operating conditions.
\end{abstract}

Keywords-AC Machine, Condition monitoring, double cage rotor, fault diagnostics, induction motor, wavelet Transform.

\section{INTRODUCTION}

Investigations on different failure modes in induction motors have revealed that $19 \%$ of the overall motor faults are related to the rotor part [1]. A detailed analysis of this type of fault can be found in [2]. They are used for applications that require high starting torque and high efficiency in steady state operation such as crushers, stirrers, or compressors. Double cage rotors are characterized by its large outer (starting) bar resistance and large inner (running) bar leakage inductance. Since double cage motors are used for loaded startups, the outer cage must handle the large starting current for the long acceleration time, with limited path for heat dissipation [3].

Cyclic electromagnetic forces, thermal stress, environmental stress, and mechanical stress associated with the large starting current, make the outer cage more vulnerable to fatigue failure. Particularly, in case of rotor outer cage broken bar, the current in the rotor bar adjacent to the faulty one increases remarkably in comparison to the nominal current. More specifically, the circulation of inter bar currents reduce the degree of rotor asymmetry, leading to a reduced sensitivity of the related sideband current components Conventionally extracted by FFT-based spectrum analysis techniques. Recently, it was proved that this sensitivity is more reduced for a double cage separate end ring rotor than for a common end ring one [4-5]. But inter bar currents produce core vibrations, which can be detected using vibration analysis techniques. The situation is more complicated when the frequency components characteristics of the rotor fault are very close to the fundamental one, and specifically under low load operating conditions [6-7]. In order to discern cases in which the presence of inter bars currents decrease the sensitivity of the MCSA, axial and radial vibrations analysis were investigated in [8], and more recently for double cage motor in [9]. On the other hand, the rotor current mainly flows in the symmetric inner cage under steady state operating condition, and fault signature is insensitive to outer cage damage. A combined use of current and vibration analysis was developed, by correlating the signal spectra to enhance broken bars detection ability under loaded and unloaded operating conditions of the motor in [10].

The fault components, issued from current or vibration signals, whose amplitudes must be monitored for diagnostic purposes, were mainly investigated in frequency domain using the classical Fourier Analysis (FA) in order to track its 
evolutions. The techniques based on FA give satisfactory discrimination between healthy and faulty conditions, but don't provide time domain information, which is mandatory for online fault detection systems. A solution was proposed in [10] to extract accurately the contribution of the fault components related to the rotor broken bar fault, in steadystate condition, by using time-frequency technique based on improved performance of wavelet analysis. Other approaches based on wavelet analysis were proposed for extracting the left sideband component during the start-up transient [5], while in [12] both the sideband components issued from stator current are investigated during a general speed transient.

In this paper, a new diagnostic index that allows extraction of rotor fault components issued from axial vibration signals, in a single frequency band is defined to improve the fault quantification procedure. A simple sliding frequency procedure in the time domain that processes the vibration signals is proposed. After the Frequency Sliding (FS) process, the contribution of the single fault component of interest is confined in a single frequency band and extracted thereby via DWT.

\section{FAULT FREQUENCY PROPAGATION}

Double cage induction motor is subjected to electromagnetic and mechanical forces symmetrically repartitioned, like any other rotating electrical machine. In healthy conditions only the fundamental frequency $f$ exists in stator currents.

If the rotor part is damaged, the rotor symmetry of the machine is lost producing a reverse rotating magnetic field related to an inverse sequence component at frequency $-s f$. This inverse sequence is reflected on the stator side, producing the frequency $(1-2 s) f$. These frequency components generate electromagnetic and mechanical interactions between stator and rotor parts. Consequently torque and speed ripple effects are generated at frequency $2 s f$, which modulate the rotating magnetic flux [2].

This modulation produces two current components, i.e., an additional left-side component at $(1-2 s) f$ and a right side component at $(1+2 s) f$. Following this interaction process, the frequency content of the stator currents show series of fault components at the following frequencies $((1 \pm 2 k s) f)_{k=1,2,3, \ldots}$. More specifically, for large or double squirrel-cage motors, as long as the contact impedance between the rotor bars and iron core is small or the copper bars are directly inserted into the laminated iron slots, the broken bar is no longer a physical condition ensuring an open circuit and inter bar or cross-path currents can flow. As a consequence, these inter bar transverse currents interact with the radial stator flux density, generating axial forces. These facts lead mainly to the presence of a first chain of fault frequency components at $\left(f_{\text {mec }} \pm 2 k s f\right)_{k=1,2,3, \ldots}$, and a second one at $((6-2 k s) f)_{\mathrm{k}=1,2,3, \ldots}$, in radial and axial vibration directions ( $f_{\text {mec }}$ denotes the mechanical speed of the motor) [7], [12]. In the next sections, the focus will be exclusively on tracking the most relevant fault components of the harmonic chains $((6-2 k s) f)_{\mathrm{k}=1,2,3, \ldots}$ and $\left(f_{m e c} \pm 2 k s f\right)_{k=1,2,3, \ldots}$, issued from axial vibrations, in frequency, and Time-Frequency domains.

\section{The Proposed TIME-FreQUENCY TECHNIQUE}

The contribution of the above listed chains characteristics of rotor fault conditions, were widely investigated in frequency domain using classical FA in order to track its evolutions. These techniques, reveals only the frequencies content of signals, but don't provide time domain information, which is mandatory for an accurate fault detection process. On the other hand wavelet transform (WT) provides greater resolution in time for high frequency components of a signal and greater resolution in frequency for low frequency components. In this sense, wavelets have a window that automatically adjusts to give the appropriate resolution developed by its approximation and detail signals. Wavelet analysis is signal decomposition, using successive combination of approximation and detail signals. The procedure is repeated until the original signal is decomposed to a pre-defined $\mathrm{J}$ level decomposition. With the well known dyadic down sampling procedure, frequency bands of each level of decomposition are related to the sampling frequency. Hence, these bands can't be changed unless a new acquisition with different sampling frequency is made. This fact complicates any fault detection based on DWT, particularly in time-varying condition.

In this paper, an efficient solution to overcome this limitation is proposed. With a sampling frequency $f_{s}=2.0 \mathrm{kHz}$, a six level decomposition $(J=6)$ was chosen in order to cover the frequency bands in which we can track the contribution of the first chain of harmonic $((6-2 k s) f)_{\mathrm{k}=1,2,3, \ldots}$. The second chain of fault component $\left(f_{m e c} \pm 2 k s f\right)_{k=1,2,3, \ldots}$, will be tracked in a frequency band corresponding to seven level decomposition $(J=6)$. The frequency band repartition corresponding to the above decompositions are detailed in Table. I.

Under healthy conditions, the fault components related to the chains $((6-2 k s) f)_{\mathrm{k}=1,2,3, \ldots}$ and $\left(f_{\text {mec }} \pm 2 k s f\right)_{k=1,2,3, \ldots}$, are lower. If the rotor part is damaged, the above rotor fault components increase significantly, and during speed-varying conditions their magnitudes are spread in a large frequency band proportional to the speed. In these conditions, the use of frequency techniques based on FA can leads to erroneous interpretations for diagnosis. In order to sense the contribution of these fault components in time-frequency domain, an optimal use of wavelet analysis is here proposed to detect rotor bar breakage with high precision. A simple processing of the axial vibration signal allows shifting the fault components to a prefixed frequency band. In such a way, all the information related to the fault is isolated and confined in a single

TABLE I.

FREQUENCY BANDS AT EACH LEVEL

\begin{tabular}{|r|l|r|l|}
\hline $\begin{array}{r}\text { Approximations } \\
« a_{j} »\end{array}$ & $\begin{array}{r}\text { Frequency } \\
\text { bands }(\mathrm{Hz})\end{array}$ & $\begin{array}{r}\text { Details } \\
\left\langle d_{j} »\right.\end{array}$ & $\begin{array}{c}\text { Frequency } \\
\text { bands }(\mathrm{Hz})\end{array}$ \\
\hline$a_{7}$ & $:[0-7.812]$ & $d_{7}$ & $:[7.812-15.625]$ \\
\hline$a_{6}$ & $:[0-15.625]$ & $d_{6}$ & $:[15.625-31.25]$ \\
\hline$a_{5}$ & $:[0-31.25]$ & $d_{5}$ & $:[31.25-62.5]$ \\
\hline$a_{4}$ & $:[0-62.5]$ & $d_{4}$ & $:[62.5-125]$ \\
\hline$a_{3}$ & $:[0-125]$ & $d_{3}$ & $:[125-250]$ \\
\hline$a_{2}$ & $:[0-250]$ & $d_{2}$ & $:[250-500]$ \\
\hline$a_{1}$ & $:[0-500]$ & $d_{1}$ & $:[500-1000]$ \\
\hline
\end{tabular}


frequency band. More in detail, a frequency sliding with $f_{s l}$ is applied at each time slice to the axial vibration signal as in (1), so that the harmonic component of interest is moved to a frequency band, in which the fault components will be tracked.

$$
V_{s l}(t)=\operatorname{Re}\left[v_{a x}(t) e^{-j 2 \pi f} s l^{t}\right]
$$

Then the real part of the shifted signal is analyzed by means of DWT. Hence, with respect to the frequency bands reported in Table I, the chains of fault components $((6-2 k s) f)_{\mathrm{k}=1,2,3, \ldots}$ and $\left(f_{\text {mec }} \pm 2 k s f\right)_{k=1,2,3, \ldots}$, will be tracked in the frequency bands [0: 15.625] and [0: 7.812] respectively.

In the next sections, frequency and Time-Frequency axial vibration analysis, under healthy and rotor broken bars, are presented and commented.

\section{ROTOR BROKEN BAR DETECTION UNDER SPEED- VARYING CONDITIONS}

In order to evaluate the performances of the proposed approach, two double cage induction motors are available; one healthy, and the second with a drilled broken bar (the bar was completely disconnected from the common end-ring). The characteristics of the double cage induction motors used for experiments are presented in Table II. A three-phase autotransformer of $30 \mathrm{kVA}, 0-380 \mathrm{~V}$ is used as motor regulated supply. A four quadrants $7,83 \mathrm{~kW}$ dc electrical drive is adopted to reach the different planned load conditions. One piezoelectric accelerometers Brüel \& Kjær model 4507 B 005, was mounted for measuring axial vibrations of the core motors. Fig. 1. shows detailed photos of the healthy and drilled rotor (left), and details of the test-bed (right). In Fig.2 and Fig.3 instantaneous values of rotor speed and axial vibration signals are depicted for healthy and faulty conditions respectively. All signals reported have been recorded during 20 seconds. The tests were carried out considering a speed transient from $0 \%$ to $70 \%$ of full load.

\section{A. Frequency domain analysis}

In this section, FFT is used to carry out a preliminary study to detect experimentally the contribution of the chain of rotor fault component $((6-2 k s) f)_{\mathrm{k}=1,2,3, \ldots}$. Fig. 4 shows the experimental spectra of the axial vibration signals, under healthy (Black) and rotor broken bar (Blue) conditions. It is clear that the contribution of the fault components related to the chain $((6-2 k s) f)_{\mathrm{k}=1,2,3, \ldots}$ is relevant. But being dependent on slip values, under speed varying conditions, the magnitudes of these fault components are spread in a bandwidth proportional to the speed variation, as mentioned in Fig. 4. Consequently, the use of Fourier transform in these conditions can lead to an erroneous diagnosis.

The same comments are valuable, for the contribution of the chain of rotor fault component $\left(f_{\text {mec }} \pm 2 k s f\right)_{k=1,2,3, \ldots}$. Fig. 5 show the corresponding spectra under healthy (Black) and rotor broken bar (Blue) conditions. In the frequency band close to the component $f_{\text {mec }}$, the spreading effect is evident for the two sidebands $\left(f_{m e c} \pm 2 s f\right)$.
TABLE II

DATA OF THE DOUBLE ROTOR CAGE MOTOR

\begin{tabular}{|l|r|r|}
\hline \multicolumn{1}{|c|}{ Data } & \multicolumn{2}{c|}{ Value } \\
\hline Rated Power & $\mathrm{kW}$ & 5.5 \\
\hline Rated stator voltage & $\mathrm{V}$ & 400 \\
\hline Rated current & $\mathrm{A}$ & 13 \\
\hline Rated frequency & $\mathrm{Hz}$ & 50 \\
\hline Rated speed & $\mathrm{rpm}$ & 2870 \\
\hline Rotor diameter & $\mathrm{mm}$ & 110 \\
\hline Axial length of the rotor & $\mathrm{mm}$ & 90 \\
\hline Air gap length & $\mathrm{mm}$ & 0.5 \\
\hline Number of stator slots & & 36 \\
\hline Number of rotor slots & & 30 \\
\hline
\end{tabular}
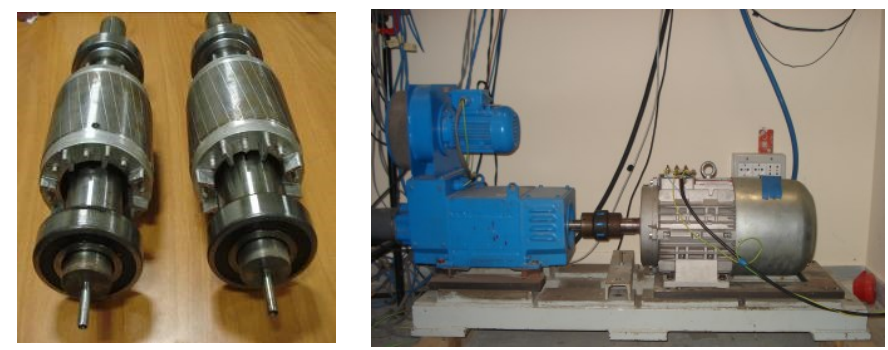

Fig. 1. Photos of the healthy and drilled broken bar (left), and details of the test-bed (right).
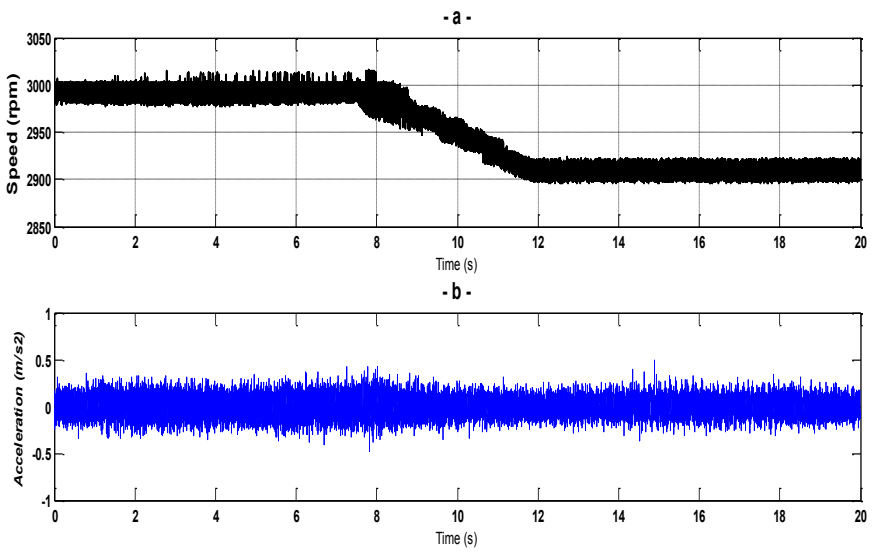

Fig. 2. Instantaneous values of speed (a), and axial vibration signal (b) under healthy conditions.
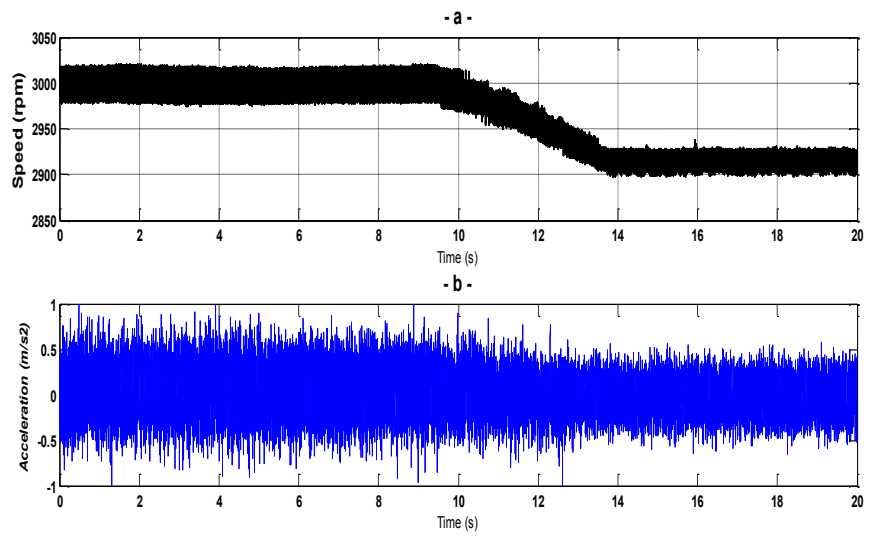

Fig. 3. Instantaneous values of speed (a), and axial vibration signal (b) under broken bar. 


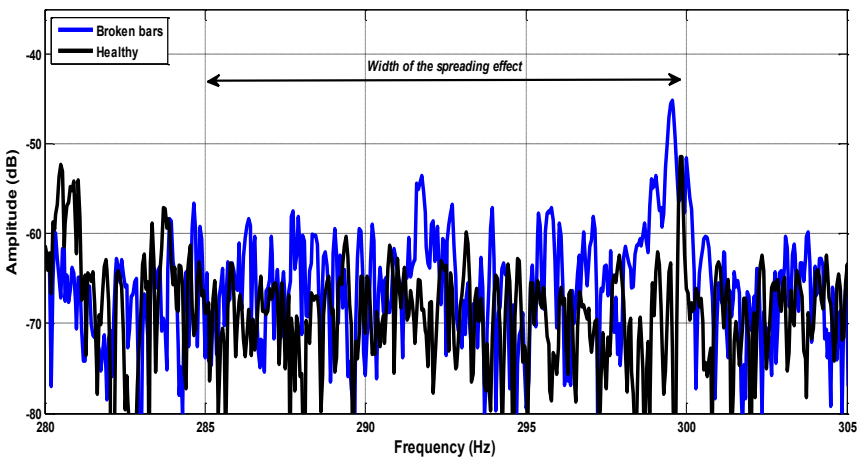

Fig. 4. Spectra of the axial vibration signals under healthy (Black) and rotor broken bar (Blue) conditions, in the range 280-305 Hz.

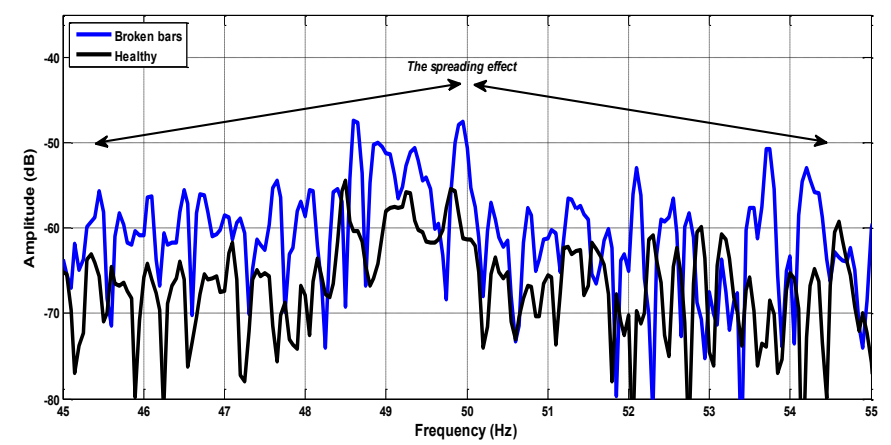

Fig. 5. Spectra of the axial vibration signals under healthy (Black) and rotor broken bar (Blue) conditions, in the range close to $f_{\text {mec }}$.

Then, a more appropriate technique is needed for providing a reliable diagnosis procedure. In the next subpart of this section, a simple and effective new technique is proposed, providing reliable information in time-frequency domain about rotor broken bar detectability in double squirrel cage induction motor.

\section{B. Time-Frequency domain analysis}

As explained in section III, the chains of fault components $((6-2 k s) f)_{\mathrm{k}=1,2,3, \ldots}$ and $\left(f_{\text {mec }} \pm 2 k s f\right)_{k=1,2,3, \ldots}$, will be tracked in the frequency bands [0: 15.625] and [0: 7.812] respectively, after appropriate frequency sliding applied to axial vibration signals. All signals reported have been recorded during 10 seconds.

The wavelet decompositions of the axial vibration signals $\left(v_{a x}\right)$ in healthy condition, for each chain or rotor fault omponents, are used as references in comparison to the faulty cases.

During the considered transient conditions, the investigated axial vibration signals under healthy and one drilled broken bar are depicted in Figs. (6-a, 8-a) and Figs. (7-a, 9-a) respectively. After a frequency sliding of $286.16 \mathrm{~Hz}$ applied to the axial vibration signals $v_{a x}$ under healthy and one rotor broken bar condition according to (1), the resulting signals $V_{s l}$ were analysed by DWT thereby. The corresponding Wavelet Analysis results $\left(a_{6}\right)$, under healthy and faulty conditions are reported in Fig. 6-b and Fig.7-b respectively.

In healthy condition, the approximation signal of interest $\left(a_{6}\right)$, depicted in Figure 6-b, shows very low variations. This indicates the absence of the chain of rotor fault component $((6-2 k s) f)_{\mathrm{k}=1,2,3, \ldots}$, leading to diagnose the healthy condition of the motor under speed-varying conditions. Under faulty condition, the contribution of the chain of fault components $((6-2 k s) f)_{\mathrm{k}=1,2,3, \ldots}$, observed on the $6^{\text {th }}$ approximation signal, is very relevant in comparison to the healthy case (Fig. 7-b). More in detail, the oscillations observed in the signal $a_{6}$, with quasi-constant amplitude, follow a characteristic pattern that fits the evolution in frequency of the considered chain of fault components. The same observations are valuable for the contribution of the chain of rotor fault component $\left(f_{\text {mec }} \pm 2 k s f\right)_{k=1,2,3, \ldots}$, observed under healthy and faulty conditions, in Fig. 8-b and Fig. 9-b respectively. The variations observed in the approximation signal $a_{7}$ are evident in faulty case (Fig. 8-b) in comparison to the healthy one (Fig. 9-b), leading to a clear discrimination between healthy and faulty conditions, through the evolution of the wavelet signal $a_{7}$.

It is worth noting that to avoid ambiguous results, the motor must be enough loaded. Anyway, the developed approach is devoted to on-line diagnosis motors, which are mostly enough loaded, making the fault detection procedure more accurate. After the above qualitative analysis of the fault, a quantitative evaluation of the fault extend was conducted in the next section, where results are commented and discussed.

\section{QuANTITATIVE Rotor BROKEn BAR EVALUATION}

Once the state of the machine is qualitatively diagnosed, a quantitative evaluation of the fault degree is necessary. For these purposes a dynamic multiresolution mean power indicator $m P a_{j}$ at different resolution levels was introduced as a diagnostic index to quantify the extent of the fault as:

$$
m P a_{j}=\frac{1}{N} \sum_{n=1}^{N}\left|a_{j}(n)\right|^{2}
$$

Where $N$ is the number of samples and $j$ is the level decomposition. In fact, the MRA analysis decomposes the signal in different frequency levels. This choice reduces the feature dimension and consequently the computational time. The fault indicator is periodically calculated (every 400 samples) using a window of 6400 samples as depicted in Figure 1, where $\delta n=150$ samples and $\Delta n=6400$ samples.

The $\mathrm{n}$ sequences are indexed by a Time Interval Number (TIN). These values were regulated experimentally to reduce variations that can lead to false alarm in healthy operating conditions of the motor. When the fault occurs, the energy distribution of the signal is changed at the resolution levels related to the characteristic frequency bands of the default. Hence, the energy excess confined in the approximation is considered as an anomaly indication in case of rotor faults.

The mean power calculation results, issued from the approximation signal $a_{6}$, which have been obtained in Figs. 6-b and 7-b, are depicted in Fig. 10. In healthy condition, the calculated $\mathrm{mPa}_{6}$ indicator don't show any significant changes. Consequently, the indicator values for the healthy motor response are considered as a baseline to set the threshold for discriminating healthy from rotor broken bar conditions. In 


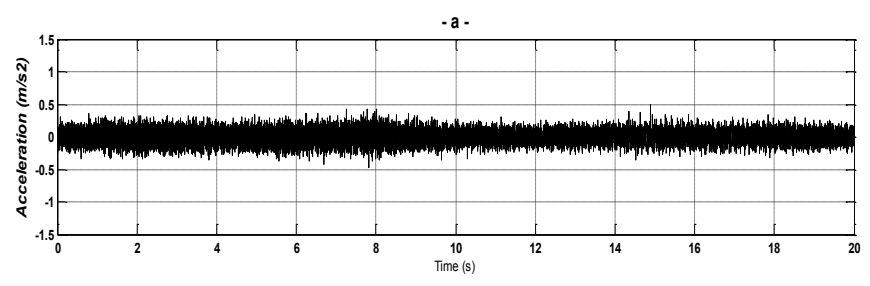

$\cdot b$.

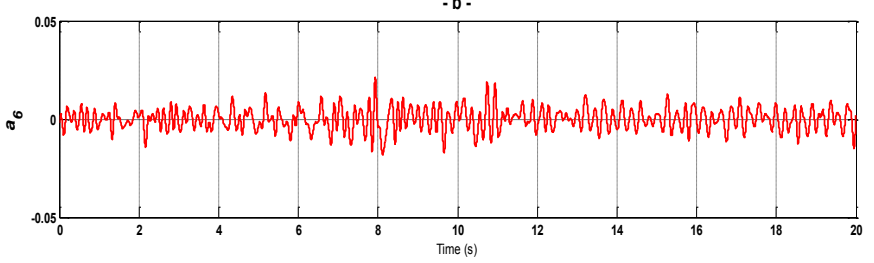

Fig. 6. Instantaneous values of axial vibration signal (a), and its corresponding Wavelet analysis (b) under healthy condition.

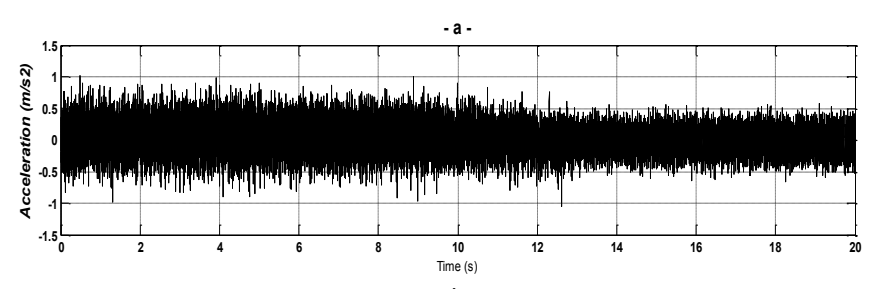

-b -

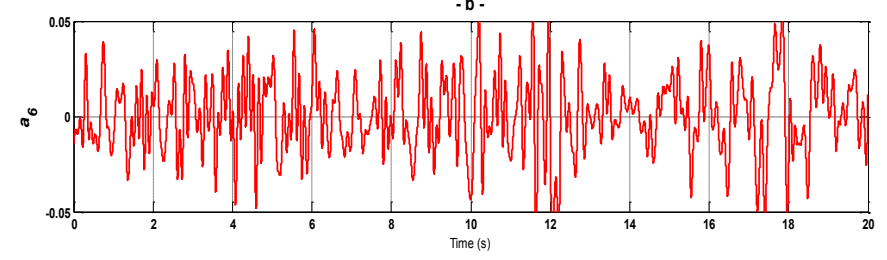

Fig. 7. Instantaneous values of axial vibration signal (a), and its corresponding Wavelet analysis (b) under rotor broken bar.

faulty conditions the calculated $\mathrm{mPa}_{6}$ indicator shows significant increase. The large energy deviation observed in faulty conditions reflect the presence of the chain of rotor fault components $((6-2 k s) f)_{\mathrm{k}=1,2,3, \ldots}$, leading to an effective, diagnosis procedure for broken bars in double squirrel cage induction machine.

The above conclusions are valid also for the quantification process related to the contribution of the chain of rotor fault components $\left(f_{\text {mec }} \pm 2 k s f\right)_{k=1,2,3, \ldots}$, and depicted in Fig. 11 for the healthy and faulty cases. Other fault components could be treated in the same way for improving the quantification process.

\section{CONCLUSIONS}

The use of classical motor current signature analysis technique may fail due to the presence of inter bar currents, which reduce the amplitude of the tracked fault components in frequency domain. Vibration analysis may not only detect the existence of inter bar currents under broken bar failure, but also enhance the detectability of this type of fault by considering classical or advanced use of signal processing techniques. For small induction motors, the MCSA diagnostic procedure is still effective as proved in [8]. But, with high power or double cage

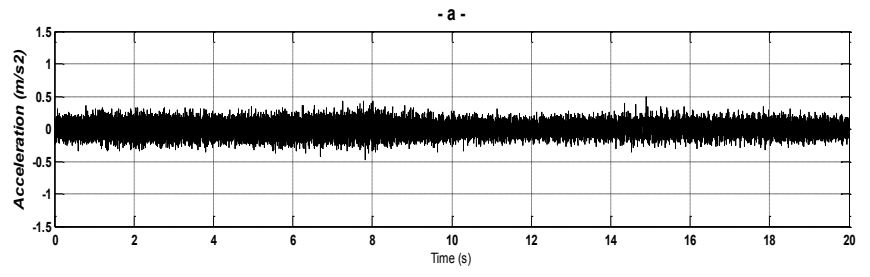

$\cdot b$.

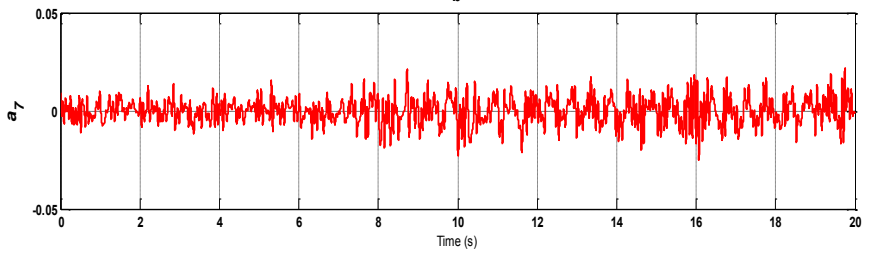

Fig. 8. Instantaneous values of axial vibration signal (a), and its corresponding Wavelet analysis (b) under healthy condition.
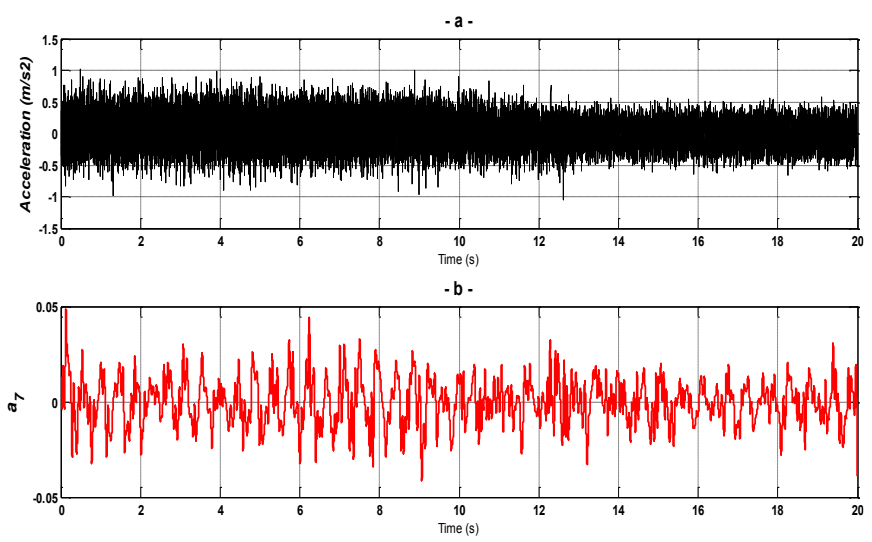

Fig. 9. Instantaneous values of axial vibration signal (a), and its corresponding Wavelet analysis (b) under rotor broken bar.

motor, like the tested one, the use of vibration analysis is mandatory in order to avoid erroneous diagnosis.

The signature issued from axial vibration analysis is relevant, for both chains of rotor fault components ((6$2 k s) f)_{\mathrm{k}=1,2,3, \ldots}$, and $\left(f_{\text {mec }} \pm 2 k s f\right)_{k=1,2,3, \ldots}$. Under low load operating conditions, the most relevant fault components become very close to the fundamental ones, which complicate the diagnosis procedure for this type of motors. Tracking these fault components in Time-Frequency domain is our main challenge in the future to guaranty a reliable rotor broken bar fault signature in a double cage induction motor.

Eventually, the developed approach based on the use of current and vibration analysis can be extended for diagnosing and classifying other rotor fault types.

\section{ACKNOWLEDGMENT}

This work was supported by SDESLab (Sustainable Development and Energy Savings Laboratory)-University of Palermo and by MIUR.

\section{REFERENCES}

[1] A.H. Bonnett, C. Yung, "Increased Efficiency Versus Increased Reliability", IEEE Ind. App. Mag., Vol. 14, Issue 1, Jan./Feb. 2008. 


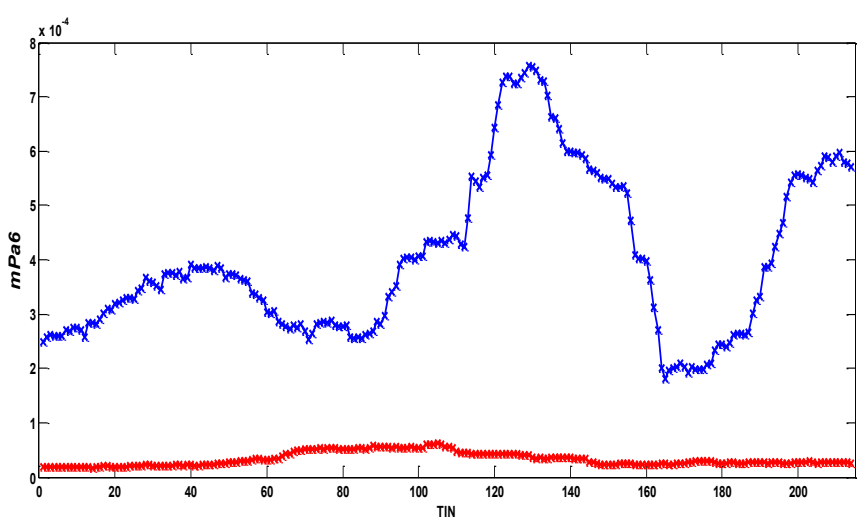

Fig. 10. Cyclic values of the $\mathrm{mPa} 6$ fault indicator calculation issued from the approximation signal a6, under healthy (Red) and rotor broken bar (Blue) conditions.

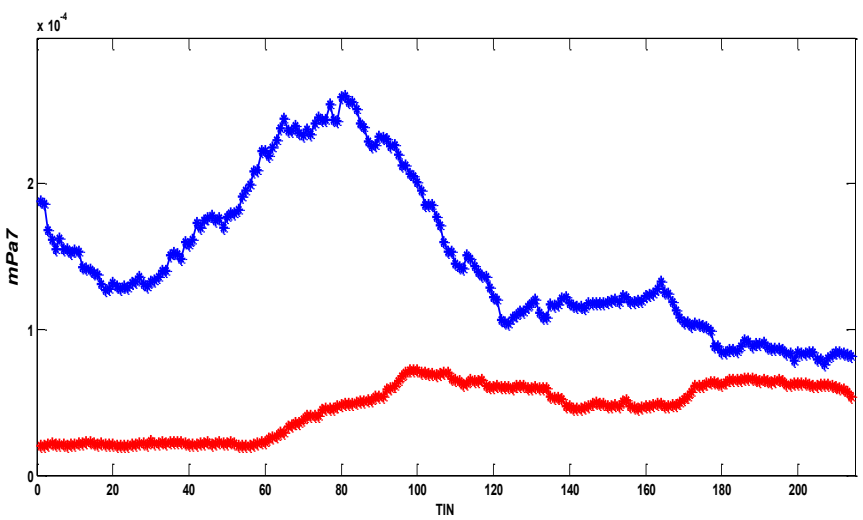

Fig. 11. Cyclic values of the $\mathrm{mPa} 7$ fault indicator calculation issued from the approximation signal a7, under healthy (Red) and rotor broken bar (Blue) conditions.

[2] A. Bellini, F. Filippetti, C. Tassoni, G. A. Capolino, "Advances in Diagnostic Techniques for Induction Machines", IEEE Tran. On Ind. Elec, vol. 55, no. 12, pp. 4109-4126, Dec. 2008.
[3] J. Mroz, "Temperature field of a double squirrel-cage motor during startup," Electric Power Applications, IEE Proceedings -, vol. 152, no. 6, pp. $1531-1538$, nov. 2005.

[4] J. Park, B. Kim, J. Yang, K. Lee, S.B. Lee, E.J. Wiedenbrug, M. Teska, and S. Han, " Evaluation of the Detectability of Broken Rotor Bars for Double Squirrel Cage Rotor Induction Motors," Proc. of IEEEECCE'10, pp. 2493-2500, Sep. 2010.

[5] J. Antonino-Daviu, M. Riera-Guasp, J. Pons-Llinares, J. Park, S. B. Lee, J. Yoo, and C. Kral," Detection of broken outer-cage bars for doublecage induction motors under the startup transient," IEEE Trans. on Ind. Appl., vol. 48, № 5, pp. 1539-1548, sept.-oct. 2012.

[6] R.F. Walliser, and C.F. Landy, "Assessment of interbar currents in double cage induction motors with broken bars," IEEE Trans. on Energy Conv., vol. 9, no. 1, pp.159-164, March 1994.

[7] A.O. Di Tommaso, R. Miceli, G. Ricco Galluzzo, "Monitoring and Diagnosis of Failures in Squirrel-Cage Induction Motors Due to Cracked or Broken Bar", IEEE-SDEMPED'2011, pp. 39-44, (Bologna) 2011.

[8] C. Concari, G. Franceschini, and C. Tassoni, "Differential Diagnosis Based on Multivariable Monitoring to Assess Induction Machine Rotor Conditions", IEEE Trans. on Ind. Elect., vol. 55, no. 12, Dec. 2008.

[9] Y. Gritli, A.O. Di Tommaso, F. Filippetti, R. Miceli, and C. Rossi, "Investigation of Motor Current Signature and Vibration Analysis for Diagnosing Rotor Broken Bars in Double Cage Induction Motors", The 21th International Symposium on Power Electronics, Electrical Drives, Automation and Motion, IEEE - SPEEDAM'2012, June 14-16, Pisa (Italy), 2010, pp. 1360-1365

[10] J. J. Rangel-Magdaleno, R. J. Romero-Troncoso, R. A. Osornio-Rios, E. Cabal-Yepez, and L. M. Contreras-Medina, "Novel Methodology for Online Half-Broken-Bar Detection on Induction Motors", IEEE Trans. on Inst. and Meas., vol. 58, no. 5, May. 2009.

[11] Y. Gritli, L. Zarri, C. Rossi, F. Filippetti, G. Capolino, and D. Casadei, "Advanced diagnosis of electrical faults in wound rotor induction machines," IEEE Trans. on Ind. Elect. (Accepted for publication), p. 1, 2012.

[12] Y. Gritli, A.O. Di Tommaso, F. Filippetti, R. Miceli, and C. Rossi "Advanced Monitoring of Rotor Broken Bar in Double Squirrel Cage Induction Machines Based on Wavelet analysis", International Symposium Ecological Vehicle Exhibition and Renewable EnergiesEVER'2012, Monaco (France), Mars 2012.

[13] A. Sadoughi, M. Ebrahimi, M. Moalem, and S. Sadri, "Intelligent Diagnosis of Broken Bars in Induction Motors Based on New Features in Vibration Spectrum", The IEEE-SDEMPED'07, pp. 106-111. Sep. 2007. 